\title{
Análisis Crítico de las Técnicas Quirúrgicas en el manejo de la Incontinencia Urinaria
}

\author{
Antonio Lomanto Morán* ; Jacinto Sánchez Angarita**
}

\section{RESUMEN}

Se presenta la experiencia de dos décadas de la Clínica de Incontinencia, en el Instituto Materno Infantil, del Departamento de Obstetricia y Ginecología de la Universidad Nacional de Colombia, de Santafé de Bogotá, sobre las técnicas quirúrgicas mas utilizadas en nuestro medio para la corrección de la Incontinencia Urinaria Femenina, haciendo énfasis en las ventajas y desventajas de cada una de ellas, complementada con toda la bibliografía mundial que existe publicada hasta la fecha sobre el tema.

Se describen las características de las técnicas vaginales, consideradas con una eficacia relativamente deficiente, pues no alcanza el sesenta por ciento de éxitos y aconsejándose su uso solamente en aquellos casos en los cuales no se disponga del conocimiento y la experiencia de otros procedimientos.

Las Infrapúbicas, que son las que fijan el cuello vesical a la cara posterior del pubis consideradas como las técnicas sugeridas cuando existe patologías distópicas vaginales, pero con una relativa disparidad estadística de resultados, pues mientras algunas escuelas reportan mas del noventa por ciento, en otras no alcanza el setenta por ciento de eficacia. El respaldo bibliográfico a nivel mundial es bajo. Las Suprapúbicas, son las que fijan la fascia vaginal a la cara posterior del pubis, ya sea al periostio o al Ligamento de Cooper. Son las de mayor experiencia, de más alta eficacia y corroborada por la literatura Mundial. Finalmente las técnicas Combinadas, son las que se practican disecando la uretra o el cuello vesical y levantándolos en lo posible con material no absorbible a la pared abdominal. Sus resultados, son buenos a corto tiempo, pero a largo plazo desciende notablemente su eficacia, pues su tasa de éxitos no alcanza el setenta por ciento

PALABRAS CLAVES: Incontinencia, Infrapúbicas, Suprapúbicas, Combinadas, Vaginales, Distópicas

\section{SUMMARY}

This Work represent the experience of two decades of work y de Departament of Obstetric and Ginecology from the Materno Infantil Hospital in Bogotá, related to the different surgical techniques used in the correction of Urinary Incontinence in Women. At the same time illustrated the adventage and disadvantage of each one of these techniques, as well as a complete revision of the world literature related to this field.

Description of the different vaginal techniques is mentioned, In it, the low response to this treatment that only shows a $60 \%$ of response is described. It is advised that this technique should only be use in cases in which the knowledge and experience of other techniques is limited.

Other techniques as is the case of the "infrapubic" technique in which the fixation of the vesical neck to the posterior face to the pubis is considered as the most suitable procedure when there is the existence of "distopic" vaginal pathologies. However, the efficiency of such procedure has been discussed since different schools shows different statistic results that ranges from $70 \%$ to $90 \%$. The revision of bibliography shows as well low results.

In contrast, the "suprapubic" technique which fix the vaginal fascia to the posterior face of the pubis (periosto or Cooper Ligament) are the procedure that need more experience but are the ones that produce the highest results, not only in our experience but at the world level.

Finally, the "mix- technique", which desiccated the urethra or vesical neck to the abdominal wall is the procedure that shows is a short time good results but the long run shows a very low efficiency since the statistic results only shows a $70 \%$ of success.

KEY WORDS: Incontinence, infrapubic, suprapubic, mix technique, vaginal technique, distopic.

Profesor Honorario. Coordinador Clínica de Incontinencia. Departamento de Obstetricia y Ginecología. Facultad de Medicina. Universidad Nacional de Colombia.
** Profesor Asociado. Docente Clínica de Incontinencia. Departamento de Obstetricia y Ginecología. Facultad de Medicina. Universidad Nacional de Colombia. 


\section{Introducción}

Las investigaciones sobre Incontinencia Urinaria Femenina en nuestro medio son relativamente fragmentarias, pues las primeras publicaciones se refieren a cirugías, cuyos resultados de aparente eficacia ensombrecen su veracidad por fallas en el seguimiento, lo cual constituye en la actualidad una información muy práctica para valorar los resultados.

Tradicionalmente se ha esgrimido que el hecho de disponer de múltiples técnicas, sugiere que ninguna de ellas sea la ideal, pero de acuerdo con la medicina basada en la evidencia, cada caso requiere de un procedimiento que generalmente se escoge de acuerdo con las experiencias personales del cirujano, con una detallada y completa información a las pacientes.

Nos proponemos a continuación presentar las experiencias realizadas durante dos décadas en el área de postgrado del Departamento de Obstetricia y Ginecología de la Universidad Nacional de Colombia, en donde en forma continua y permanente se han desarrollado los programas de Ginecología Urológica, dirigidos específicamente a la Patología de la Incontinencia Urinaria Femenina.

Estos programas han sido desarrollados con un grupo integral de especialidades, en donde han participado las áreas de Urología, Imágenes Diagnósticas, Psicología, Fisioterapia, Trabajo Social, Nutrición y Endocrinología, cuyos criterios y conductas han sido reformadas no solo de acuerdo con las experiencias obtenidas en el servicio, sino complementada con la investigación bibliográfica actualizada por el personal de post- grado y supervisada por los docentes de la Clínica

Sin lugar a dudas, las técnicas perfectamente concebidas y ejecutadas por profesionales de experiencia en la corrección de la Incontinencia Urinaria Femenina, han comprobado una proporción de fracasos, y así el análisis de estos han dado pautas verdaderamente significativas de enseñanza para tener en cuenta en procedimientos futuros.

Aún existe mucha incertidumbre acerca del procedimiento ideal que otorgue la mejoría definitiva de esta patología, con las técnicas descritas hasta el momento, pues las estadísticas entre los diferentes países discrepan notablemente.

\section{Criterios Básicos Para La Cirugía}

En la actualidad son múltiples los meta-análisis y más de cinco mil artículos publicados sobre las diferentes técnicas, pero de todo este material solamente un porcentaje muy bajo de los artículos cumplen con los requisitos mínimos epidemiológicos, con los que se pueden obtener resultados para concluir cuales son las técnicas más adecuadas para el manejo quirúrgico de la Incontinencia Urinaria Femenina.

Con éste orden de ideas, podemos enumerar algunos puntos considerados como fundamentales para obtener el mayor número de posibilidades de éxito con las técnicas quirúrgicas:

- Anamnesis completamente detallada, haciendo énfasis en la patología gineco-urológica.

- Examen físico, realizado con criterios muy bien establecidos.
- Estudios de laboratorio orientados a definir con claridad patologías relacionadas con cambios hormonales y sistémicos.

- Pruebas correctoras de la sintomatología, con el fin de restablecer la ubicación de las estructuras anatómicas a su sitio original.

- Procedimientos paraclínicos específicos, vale decir como el patrón de oro: la Urodinamia.

- Selección de la Técnica de acuerdo a la evaluación clínica.

- Capacidad tanto en el área cognoscitiva como de habilidades y recursos por parte del cirujano.

- Información pormenorizada a la paciente del tipo de intervención, así como riesgos, complicaciones y posibilidades de eficacia de la técnicas.

\section{Objetivos Fundamentales de las Técnicas}

Han sido innumerables las teorías publicadas que reporta la literatura mundial, sobre la fisiopatología de los procedimientos quirúrgicos para la corrección de la Incontinencia Urinaria Femenina, pero en la actualidad se han limitado a los siguientes aspectos:

- De acuerdo con los principios de Ingeniería Biomédica, es muy importante preservar la movilidad de la uretra con lo cual facilita la funcionalidad y así recuperar la continencia.

- Teniendo en cuenta, la íntima relación de los fascículos musculares del elevador del ano con los mecanismos de continencia, es de gran importancia practicar la recuperación del piso pélvico.

- Posiblemente el objetivo mas importante de las técnicas, se fundamenta en la ubicación del cuello vesical y la uretra proximal intraabdominal.

Además de los conceptos emitidos anteriormente, en lo que respecta a los criterios básicos de las técnicas, consideramos que es altamente preocupante lo que se relaciona con la micción postoperatoria, pues de todos es conocido que el catéter suprapúbico, recurso poco utilizado en nuestro medio, es sin lugar a dudas el método ideal, pero ante la dificultad de su uso, hemos empleado la sonda transuretral el menor tiempo posible, logrando en nuestro servicio como tiempo mínimo cuarenta y ocho horas

\section{Técnicas Vaginales}

Indudablemente son las técnicas más antiguas descritas en la literatura médica, que fueron muy aceptadas hace cuatro o cinco décadas, con gran disparidad de resultados, posiblemente por la dificultad en la aplicación de los procedimientos urodinámicos que aún no existían en esa época.

Otra circunstancia que pudo incidir en los resultados, fue el hecho de definir el tipo de intervención de acuerdo con las medidas comparativas entre la base de la vejiga y la uretra, criterios totalmente revaluados en la actualidad por tratarse de conceptos de carácter estático.

Las técnicas, se fundamentan en la suspensión de la uretra y el fruncimiento del cuello vesical, ubicándolo preferiblemente sobre el plano vertical bajo. Infortunadamente de acuerdo con los criterios aceptados a nivel internacional, 
no cumple con los requisitos de ubicar el tercio proximal de la uretra intraabdominal, y por lo tanto no es totalmente eficaz para la corrección de la incontinencia.

El hecho, de practicar cirugías simultáneamente cuando existen distopias vaginales, y su baja estancia hospitalaria por la poca morbilidad, constituyen argumentos favorables para defender el procedimiento quirúrgico. La mayoría de las mujeres que presentan este tipo de patología son pacientes de alta paridad, cuyos partos en un gran porcentaje, no han sido atendidos adecuadamente y es lógico suponer, que las estructuras anatómicas que suspenden el cuello vesical por el trauma obstétrico, quedan lesionadas y es obvio deducir que son estructuras que no se pueden utilizar posteriormente como medio de sostén para la corrección de la incontinencia.

Otros aspectos importante que inciden en el éxito de la cirugía, son los factores nutricionales, los cambios de las concentraciones del colágeno, principalmente en las perimenopáusicas, y el criterio recientemente corroborado por las investigaciones como es el factor racial. En relación a este último, las mujeres de raza negra tienen una menor prevalencia de patología relacionada con la incontinencia urinaria.

Con las investigaciones más recientes, es importante anotar que el hecho de comprometer el cuello vesical durante la técnica, es un recurso desfavorable, pues de acuerdo con las publicaciones basadas en la Ingeniería Biomédica, tanto el cuello vesical como la uretra deben conservar su movilidad para preservar la continencia. Cuando se han practicado intervenciones vaginales, y se intenta realizar la disección de esas estructuras, se corre el riesgo de ocasionar soluciones de continuidad tanto en la uretra como en la vejiga y con una alta morbilidad postoperatoria.

Las diferentes escuelas, preocupadas por la baja eficacia, en las últimas décadas han apelado a diferentes aditamentos de carácter artificial ubicándolos tanto en la uretra como en el cuello vesical, lo que al parecer no ha logrado modificar los resultados.

El empleo de material no absorbible ha sido motivo de discusiones, criterio que puede ser fácilmente cuestionado, pues según las normas elementales de técnica quirúrgica, la cicatrización no se realiza adecuadamente con este tipo de material, ya que está fundamentada principalmente en la irrigación y en las adherencias posteriores que se realizan en esas zonas cruentas.

Inicialmente las publicaciones obtenidas de la literatura reportaban una aceptable eficacia, pero con un sesgo altamente significativo, que era el hecho de no consignar el seguimiento a largo plazo, lo cual riñe con las normas más elementales de los criterios epidemiológicos.

En conclusión, después de revisar minuciosamente la literatura mundial, y basados en la experiencia de nuestro servicio, la eficacia de los procedimientos vaginales nunca superan a las otras técnicas para la corrección de la Incontinencia Urinaria Femenina, por lo cual sugerimos que la única razón para practicarla sería en aquellos casos en los cuales no se dispone de los conocimientos y habilidades para realizar otras técnicas similares.

\section{Técnicas Suprapúbicas}

Con las vaginales, han logrado el mayor número de experiencias publicadas en la literatura mundial, y han sido objeto de múltiples modificaciones con el propósito de obtener menos efectos colaterales y una eficacia mayor a largo plazo.

De todas las técnicas practicadas en nuestro servicio, los procedimientos suprapúbicos son las intervenciones con las cuales tenemos más experiencia, y de cuyos resultados haremos énfasis más adelante.

Son las que se practican por vía abdominal, utilizando estructuras anatómicas como la fascia vesicovaginal y en algunas ocasiones fijándolas con materiales sintéticos y con el propósito de ubicar el cuello vesical intra-abdominal, en lo posible sin perforar la mucosa vaginal, fijándola al periostio retropúbico o estructuras mas altas como el ligamento de Cooper.

Somos partidarios de las incisiones paralelas al arco púbico, y respetando los rectos abdominales, con el propósito de obtener un campo quirúrgico adecuado. Posiblemente no son consideradas como las incisiones más fisiológicas, pues se comprometen las estructuras anatómicas en sentido transversal, pero también es cierto que son las que presentan menos posibilidades de eventraciones post-operatorias.

Un argumento esgrimido por algunos autores, es el hecho de que una zona muy vascularizada como es el espacio de Retzius, en el cual hay una alta posibilidad de que se presenten hematomas, sugieren la necesidad del uso de drenajes en el post-operatorio.

De acuerdo con nuestra experiencia el recurso del drenaje, ha sido utilizado en situaciones excepcionales cuando es muy evidente el sangrado en capa, y cuando ha sido muy difícil completar la hemostasia. En lo posible tratamos de no utilizarlos, por que somos conscientes de la alta frecuencia de infecciones que conlleva su uso.

En las Técnicas Suprapúbicas, las estructuras son fáciles de identificar y las zonas de anclajes son muy resistentes, como periostio y el Ligamento de Cooper. Cuando se fijan al periostio, hemos comprobado entre el uno y el cinco por ciento de osteítis asépticas postoperatorias, complicación que ocasiona mucho dolor y su tratamiento requiere de medicamentos y fisioterapia, caracterizado por una recuperación relativamente lenta.

En lo referente al tipo de suturas, existe una controversia sobre la utilidad con las "no absorbibles" a largo plazo, ya que en las publicaciones mas extensas reportadas hasta la fecha, las cirugías son practicadas con material absorbible a "mediano plazo". En nuestro servicio como norma, siempre hemos utilizado material no absorbible y en una revisión preliminar realizada recientemente en un estudio comparativo, aun no publicado, el uso de este material ha sido muy favorable a largo plazo.

$\mathrm{El}$ enterocele es una complicación relativamente frecuente, que oscila entre el ocho y el quince por ciento, patología muy explicable, pues al "levantar "las estructuras anteriores distorsiona las relaciones anatómicas, y tracciona el piso pélvico hacia delante.

Algunos autores, teniendo en cuenta ese problema, con una actitud considerada como profiláctica, aunque no 
muy académica, practican durante el acto quirúrgico el cierre del fondo del Saco de Douglas. No existen hasta la fecha publicaciones que respalden éste ultimo procedimiento, para darle validez estadística.

Los procedimientos suprapúbicos, tienen una morbilidad relativamente alta, lo que conlleva una mayor estancia, criterio desfavorable en nuestro medio por los altos costos hospitalarios.

En nuestro servicio, después de casi dos décadas de experiencia con estos procedimientos, y teniendo en cuenta que su eficacia supera el noventa por ciento, la consideramos como la más aconsejable de las técnicas para el manejo de la Incontinencia Urinaria Femenina, siempre y cuando se cumplan los requisitos anteriormente expuestos

\section{Técnicas Infrapúbicas}

De todo el grupo son las técnicas de más reciente publicación y obviamente son pocas las experiencias que reportan seguimiento a largo plazo.

Consisten, en incisiones vaginales y después de identificar el cuello vesical se fija con un punto a cada lado en la cara posterior del pubis, con material no absorbible.

Llama la atención que son técnicas prácticamente desconocidas en los países anglosajones y en Europa, y las pocas publicaciones que existen figuran solamente las del autor, con seguimientos tan reducidos, que no cumplen con los requisitos mínimos internacionales para considerarles con eficacia aceptable.

El Departamento de Obstetricia y Ginecología de la Universidad de El Bosque, tiene la mayor experiencia, con un seguimiento mayor de diez años y una eficacia por encima del noventa por ciento.

En nuestro servicio, figura dentro de las técnicas ideales cuando a la Incontinencia se suman relajaciones del piso pélvico importantes, y hasta la fecha los resultados aún no son representativos pues no cumplen con el tiempo ideal para establecer su eficacia

Tenemos que aceptar que es una técnica que requiere el recurso de habilidades y destrezas, en lo posible con una casuística mucho más amplia, pues en algunos hospitales universitarios, posiblemente por fallas en el procedimiento y ante los resultados no satisfactorios, han desistido de continuar utilizándola.

Es sin lugar a dudas, la más representativa del grupo que se constituiría en el procedimiento de elección, cuando la mujer presenta distopias útero-vaginales asociadas a Incontinencia Urinaria de Esfuerzo, relativamente sencilla para los especialistas que trabajan en el área ginecológica y cuando es practicada con las todas las especificaciones es posiblemente la mas rápida de todas las que hemos descrito.

Teniendo en cuenta que la técnica es completamente vaginal, las complicaciones postoperatorias son similares a las que se hacen por esa vía con una estancia hospitalaria relativamente corta, lo que se constituye en una característica altamente favorable en nuestro medio.

De acuerdo con las normas establecidas en el estudio de la Incontinencia Urinaria de Esfuerzo, en el sentido de que el propósito fundamental de las técnicas es ubicar el cuello vesical y el tercio proximal de la uretra intraabdominal, podemos afirmar que la uretrocistopexia infrapúbica, aún con las técnicas más depuradas y en manos de expertos no alcanza a cumplir con este requisito, lo que sería desde el punto de vista teórico un argumento de mucho valor para demeritar la eficacia del procedimiento.

Otra característica que es importante consignar, es la fijación que se hace del cuello vesical a la cara posterior del pubis, limitando la movilidad del mismo, lo que riñe con los conceptos de Ingeniería Biomédica, que han demostrado el valor que tiene la fijación del cuello en lo referente a la Continencia. Además, es relativamente frecuente que por el hecho de utilizar el periostio, se presenten osteitis asépticas, de cuyo manejo comentamos anteriormente.

En conclusión, la uretrocistopexia infrapúbica, es considerada como una alternativa quirúrgica, cuando la Incontinencia está asociada a distopias tanto del útero como de las paredes vaginales anteriores y posteriores, siempre y cuando el cirujano tenga los suficientes conocimientos y experiencia.

\section{Técnicas Combinadas}

Son aquellas que después de identificar el cuello vesical por vía vaginal, logra fijarlo y suspenderlo con material no absorbible a los rectos abdominales, bajo visión endoscópica.

Las primeras técnicas, fueron descritas a principio de este siglo y con el transcurso del tiempo, han sido objeto de múltiples modificaciones, como el utilizar cintas homólogas o de carácter sintético, que levantando el cuello lo fijan posteriormente a los rectos. Este tipo de apoyo ha sido criticado por los detractores de la técnica, pues consideran que no posee la suficiente resistencia para conservar la continencia a largo plazo.

Inicialmente la fijación y ascenso del cuello se realizó en forma "ciega", con agujas y posteriormente se le adiciona la endoscopia, mediante la cual no solo se logra evaluar la integridad tanto de la uretra, el cuello y la vejiga, sino que además se puede establecer exactamente la ubicación cervical. No obstante, considerar ésta última característica como una ventaja de la técnica, es relativamente alta la incidencia de retenciones urinarias en el postoperatorio.

Es un procedimiento rápido, aún si se considera como inconveniente el hecho de utilizar la endoscopia, lo que constituye una razón para que el Ginecólogo que no tiene la suficiente experiencia para practicarla, necesite el concurso del Urólogo. Una ventaja adicional, es el hecho de que se puede utilizar cuando existen patologías relacionadas con relajaciones del piso pélvico.

En la descripción inicial de la técnica, se usa como sistema de drenaje vesical la cistostomía suprapúbica, procedimiento no muy aceptado en nuestro medio, por el hecho de ser invasivo al perforar la vejiga. En nuestra experiencia, publicada hace varios años, se redujo notablemente la infección urinaria postoperatoria, facilitó el control del residuo vesical, reduciendo en ésta forma la estancia hospitalaria.

Posiblemente por el uso de la endoscopia, el riesgo de lesionar tanto la uretra como la vejiga y el rechazo como hemos dicho en nuestro medio de la cistostomía, no ha 
sido muy aceptada por la gran mayoría de los ginecólogos, que prefieren las infrapúbicas, que tienen menos riesgos.

Cuando se adquiere suficiente experiencia, tiene relativamente pocas complicaciones intraoperatorias. Sin embargo, por la ubicación del cuello vesical, es frecuente observar retención urinaria y dolor por la fijación que se hace en la pared abdominal.

No obstante las anteriores observaciones, es la técnica sugerida en aquellos casos de recidivas, antecedentes de cirugías previas, irradiaciones y en pacientes con gran panículo adiposo, que dificultan notablemente los procedimientos por vía abdominal.

Un requisito muy importante para realizar la cervico suspensión endoscópica, constituye el hecho de que la pared abdominal debe ser de buena consistencia, pues es el soporte mas importante, para sostener el cuello vesical y obviamente conservar la continencia.

Es pertinente anotar, que la técnica descrita inicialmente, utiliza en el sitio de soporte del cuello vesical, un centímetro de dacrón para que el material de sutura que lo levanta no lo "corte" al practicar el ascenso. Teniendo en cuenta que las pacientes que fueron objeto de nuestro estudio, provienen de un nivel socio-nutricional relativamente bajo, y con el agravante de que sus estructuras anatómicas no son de la consistencia ideal, resolvimos utilizar en los sitios de soporte de la pared abdominal, también material sintético para evitar ser desgarrada.

No obstante los inconvenientes que hemos anotado de la cervicosuspensión endoscópica, no podemos desconocer que con el riesgo que conlleva la lesión de estructuras tan importantes como la uretra y la vejiga, y aún con el uso de la endoscopia, se puede afirmar que es la única técnica que permite ver la ubicación del cuello, para comprobar que se encuentra intra-abdominal.
En este grupo existen suficientes publicaciones, para concluir que la cervicosuspensión endoscópica, es efectiva a determinado tiempo, pero a largo plazo la eficacia desciende notablemente.

Hace menos de una década, en algunos países se está utilizando la técnica descrita como cabestrillo (o hamacas), y que consiste en pasar elementos homólogos o de carácter sintético no absorbibles por debajo del cuello vesical, para fijarlos posteriormente a la pared abdominal, constituyéndose en uno de los pocos procedimientos ideales para corregir la incontinencia cuando existe compromiso del mecanismo esfinteriano.

Las técnicas tipo TVT, descritas hace menos de diez años y que consisten en colocar por vía vaginal una malla de polipropileno, en el tercio medio de la uretra y con unas guías la deja suspendida a la pared abdominal, finalizando el procedimiento con revisión de la vejiga y uretra con citoscopia. El autor de la técnica la sugiere para aquellos casos con grandes factores de riesgo, utilizando anestesia local.

Las publicaciones conocidas, hasta el momento están cumpliendo con el tiempo exigido a nivel internacional, para sacar conclusiones definitivas sobre la eficacia del procedimiento.

Con este artículo, se presentan los criterios que hasta el momento existen de la Medicina Basada en la Evidencia de las Conductas, en el Manejo Quirúrgico de la Incontinencia Urinaria Femenina y complementada con las experiencias del área de la Ginecología Urológica, orientada específicamente a la Incontinencia Urinaria, durante mas de veinte años, en el Departamento de Obstetricia y Ginecología de la Universidad Nacional de Colombia.

\section{BIBLIOGRAFIA}

1. Aanestad O. Et Al Urinary Incontinence update: old traditions and new concepts. Adv. Intern. Med. 1999.

2. Appleby S. et al Stress Urinary Incontinence Wound Mange - Jan 1999: 45(1): $44-8$.

3. Arenas Buenahora, Cols. La Cistopexia modificada Rev. Col. De Obs. Y Gin. 1985; 36(1): 11 .

4. Becerra, E. Evaluación de la Cistouretropexia Retropúbica Rev. Col. Obs. Y Gin. 1985, 36(6): 380.

5. Beltran. A., Incontinencia Urinaria de Esfuerzo en la mujer Rev. Col. Obs. Y Gin. 1961; 12:108.

6. Bernal. E., La uretrovesicosuspensión de Marshall-MarchettiKrantz en la corrección de la incontinencia Urinaria de Esfuerzo. Rev. Col. Obs. Y Gin. 1964; 15:159.

7. Blanc, E. et al Value of the use of a questionaire in the evaluation of incontinence surgery. Prog. Urol. 1999, febr. Abs. Avail.

8. Buzalov S. Et,al. The late results of the surgical treatment of stress urinaryoncontinence. Ak. Gyn. Soff; 1998 Abs. Av.

9. Burch, J.C., Urethrovaginal fixation to cooper ligament for correction of stress incontinence, cystocele and prolapse. Am. Jou. Obst. \& Gyn. 1961; 81: 281.

10. Diokno AC. et al. Office based criteria for procedicting type II stress incontinence without further evaluation studies. Jour. Uology, 1999; april. Abs. Avail.

11. Dwyer PL, et al, Suture Injury tothe urnary trac in urethral suspension procedures for stress incotinence, Internat, Urogynecol, J. Pelvic Floor, Disfunc; 1999 april.
12. Enhorning. et al. Uretharl Closure Studient with cine roenganoography-Aand simultaneos bladder-urethra pressure recording. Sugery. Gyn and Obs.1964; 118: 507-616.

13. Hadley. Hr., et al- The Uretral Sling And Stress Urimary Incontinence, West-Jorr-Med. 1998 Sept. 169(3): 167-8.

14. Hirsch. A. Et, al Treatment of female urinary incontinence with EMG-Controlled biofeedback home training.Intern.Urogynecol. J.Pelvic Dloor Dysfunct, 1999; abril Ab. Av.

15. Howfllin., et al Treatment of Urinary Stresss Incontinence Ann. Chir. 1999; 53(4): 32H - 34.

16. Hutchines., A. et al- Surgery for stress Incontinence Br. Jou. Urol. 1998; Nov: 82(5): 634-41

17. Kelly, H.A. Incontinence of urine in woman. Urol. And Cuta Rev. 1913; 17: 219-293.

18. Khader et. Al,Bologne procedure in stress urinary incontinence with stage III cystocele. Prog.Urol. 1.999, feb Ab. Av-

19. Liu CY, Laparoscopic treatment of Stress urinary incontinence. Obstet.Gynecolo. Clin. North. Am. 1999, marz

20. Lomanto A.: Gómez. P. Operación de Kelly, Rev. Col. Obst. Y Gin. 1983 34, 28.

21. Lomanto, A. Incontinencia Urinaria de Esfuerzo en la mujer. Tratamiento. Memorias Primeras Jornadas de Ginecología y Obstetricia. Clínica Fray Bartolomé de las Casas. Bogotá, 1984; 111.

22. Lomanto, A., Evaluación de la Colposistouretropexia en la Incontinencia Urinaria de Esfuerzo. Rev. Col. Obst. Y Gin. 1982; $32,112$.

23. Lomanto, A.: Sánchez, J. Análisis de fracasos del manejo de la incontinencia urinaria de esfuerzo. Trabajo inédito 1989. 
24. Lomanto, A.: Sánchez, J.Enfoque actual del manejo de las técnicas ., Rev. Col. Obst. Y Gin1992; 43(4): 265.

25. Lomanto, A.: Sánchez, J. La Cervicosuspensión endoscópica en el tratamiento de la incontinencia urinaria de esfuerzo. Informe preliminar Rev. Col. De Obst. Y Gin. 1987; 38(5): 331-345.

26. Lomanto, A.: Sánchez, J. Experiencia de 100 casos con la cervicosuspensión endoscópica. 1989. Trabajo de Promoción. Universidad Nacional de Colombia. En Impresión.

27. Lopez, L.A.; Corzo, J,E. Corrección de la incontinencia urinaria de esfuerzo por medio de la Uretrocistopexia retropúbica por vía vaginal Rev. Col. Obst. Y Gyn. 1983; 34, 34.

28. Low .J.A. The management of anatomic urinary incontinence by vaginal repair. Ame. Jou. Of Obst-Gyn. 1967; 97: 308-315.

29. Miller JM, et al, On the lack of correlation between self -report and urine loss measured with standing provocation test in older stress incontinence women.Jour.Wom.Heal..1999, mar Abs Av.

30. Neerajkohli et all. Count. Obs. - Gyn. May 1999.

31. Packey, K. Pathology and repair of genital prolapse, Obs-Gyn. 1949; 56: 1-15.

32. Pereyra. A.J.; Lebertz, T.B. Combined Uretrovesical Suspension and Vagiino-Urethroplasty for correction of Urinary stress Incontinence. Obst. And Gyn. 1986-87; 30: 537-546.

33. Powel, L.C.; J.R. Retropubic urethrocystopexy: Vaginal approach. Am. Jou. Obs. And Gin. 1981; 140: 91.

34. Redondo, H.; Cruz, R. Estudio clínico y enfoque terapeútico de la incontinencia urinaria de esfuerzo en la mujer. Rev. Col. Obst. Y Gin. 1986, 37.

35. Redondo, H.; Cruz, R. Uretrocistopexia Infrapública. Rev. Col. Obst. Gin. 1986, 39.
36. Redondo, H.; Lomanto. A., Incontinencia Urinaria de Esfuerzo: Revisión de la Operación Kelly en 10 años del Instituto Materno Infantil de Bogotá. 1981, Trabajo Inédito.

37. Roos, R.A. et al. Vaginal prolapse and stress urinary incontinence West Virginia Medical Journal of Obst-Gyn 97: 308-315.

38. Schmidt. R. A. Et al Urinary Incontinencia update adu. Int. Med. 1999, 44: 19-57.

39. Stamey, T.A. Endoscopic suspensión of vesical neck urinary incontinence. Surg. Gyn. Obst. 1973, 136: 547, 554.

40. Stamey, T.A. Endoscopic suspensión of the vesical neck for urinary incontinence in females, report on 203 consecutive patients. An. Of Surg. 1980, 192 (4): 465-471.

41. Stamey, T.A.; Scheffer, A. J.; Condoy, C. Clinical and roentgenographic evaluation of endoscopic suspensión of vesical neck for urinary incontinence. Sug. Gyn. Obst. 1975. 140: $355-360$.

42. Stamey, T.A. Urinary incontinence in the female Campbell Urol. 1979, 3: 2272-2293.

43. Tanagho, E.A., and. Cols. L a Cistopexia modificada Rev. Col. De Obst. Y Gin. 1976, 410: 475.

44. Tobon, G. Cols. Tratamiento de la incontinencia urinaria de esfuerzo por la operación de Burch. Rev. Col. De Obst. Y Gin. 1981, 32(1): 59.

45. Ulmsten - TVT Scant. Jour. Urol. Nefre. Nov. 1993

46. Ulmsten - TVT Brit. Jour. Obs. - Gyn. Abril 1999. Vol. 106 P: 345-350.

47. Wyman JF et al - Comparative Eficacy of Behaviora Lin. The Management of Female Urinary AM. Jou. Obst. Gyn. - 1998. Oct: 179(4): 999 - 1007.

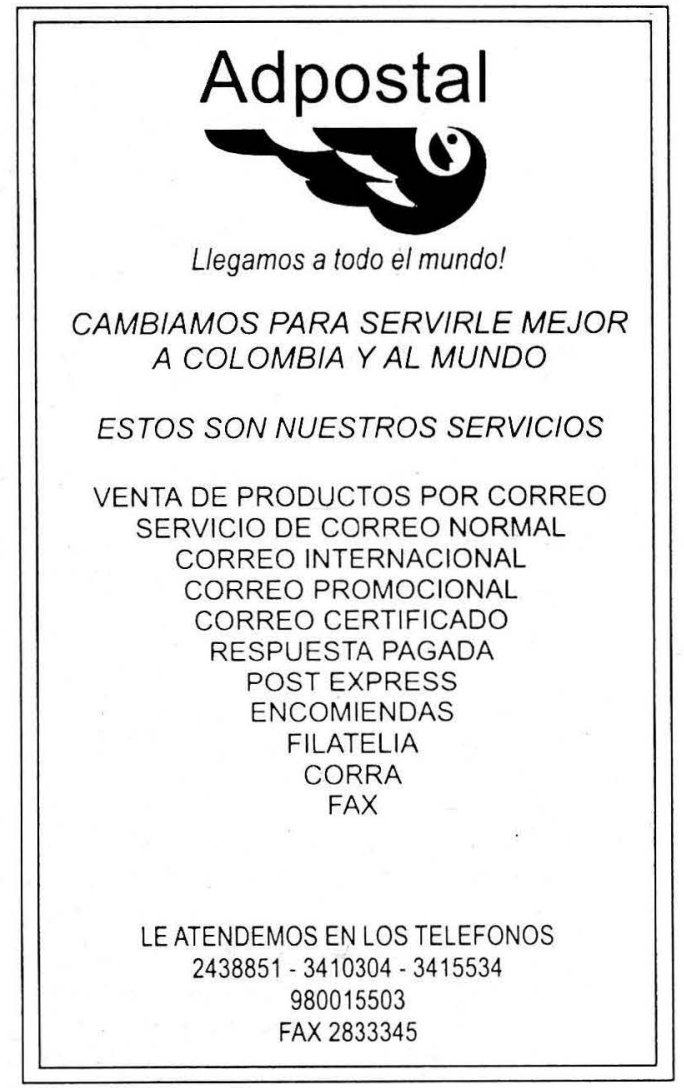

